\begin{tabular}{|c|c|}
\hline Title & Mathematical model for self-propelled droplets driven by interfacial tension \\
\hline Author(s) & $\begin{array}{l}\text { Nagai, Ken H.; Tachibana, Kunihito; Tobe, Y uta; Kazama, Masaki; Kitahata, Hiroyuki; Omata, Seiro; Nagayama, } \\
\text { Masaharu }\end{array}$ \\
\hline Citation & $\begin{array}{l}\text { Journal of chemical physics, 144(11), } 114707 \\
\text { https://doi.org/10.1063/1.4943582 }\end{array}$ \\
\hline Issue Date & $2016-03-21$ \\
\hline Doc URL & http:/hdl.handle.net/2115/64751 \\
\hline Rights & $\begin{array}{l}\text { () } 2016 \text { A IP Publishing LLC. The following article appeared in J. Chem. Phys. 144, 114707, } 2016 \text { and may be found at } \\
\text { http://scitation.aip.org/content/aip pournal /cp/144/11/10.1063/1.4943582. }\end{array}$ \\
\hline Type & article \\
\hline File Information & 1.4943582.pdf \\
\hline
\end{tabular}

Instructions for use 


\section{AlP $\mid \begin{aligned} & \text { The Journal of } \\ & \text { Chemical Physics }\end{aligned}$}

\section{Mathematical model for self-propelled droplets driven by interfacial tension}

Ken H. Nagai, Kunihito Tachibana, Yuta Tobe, Masaki Kazama, Hiroyuki Kitahata, Seiro Omata, and Masaharu Nagayama

Citation: The Journal of Chemical Physics 144, 114707 (2016); doi: 10.1063/1.4943582

View online: http://dx.doi.org/10.1063/1.4943582

View Table of Contents: http://scitation.aip.org/content/aip/journal/jcp/144/11?ver=pdfcov

Published by the AIP Publishing

\section{Articles you may be interested in}

Numerical study on the effects of non-dimensional parameters on drop-on-demand droplet formation dynamics and printability range in the up-scaled model

Phys. Fluids 24, 082103 (2012); 10.1063/1.4742913

Self-propelled motion of a fluid droplet under chemical reaction

J. Chem. Phys. 136, 074904 (2012); 10.1063/1.3685805

Spontaneous mode-selection in the self-propelled motion of a solid/liquid composite driven by interfacial instability

J. Chem. Phys. 134, 114704 (2011); 10.1063/1.3567096

Numerical simulations of interfacial instabilities on a rotating miscible magnetic droplet with effects of Korteweg stresses

Phys. Fluids 17, 042101 (2005); 10.1063/1.1870017

A model for large deformation of an ellipsoidal droplet with interfacial tension

J. Rheol. 47, 659 (2003); 10.1122/1.1562152

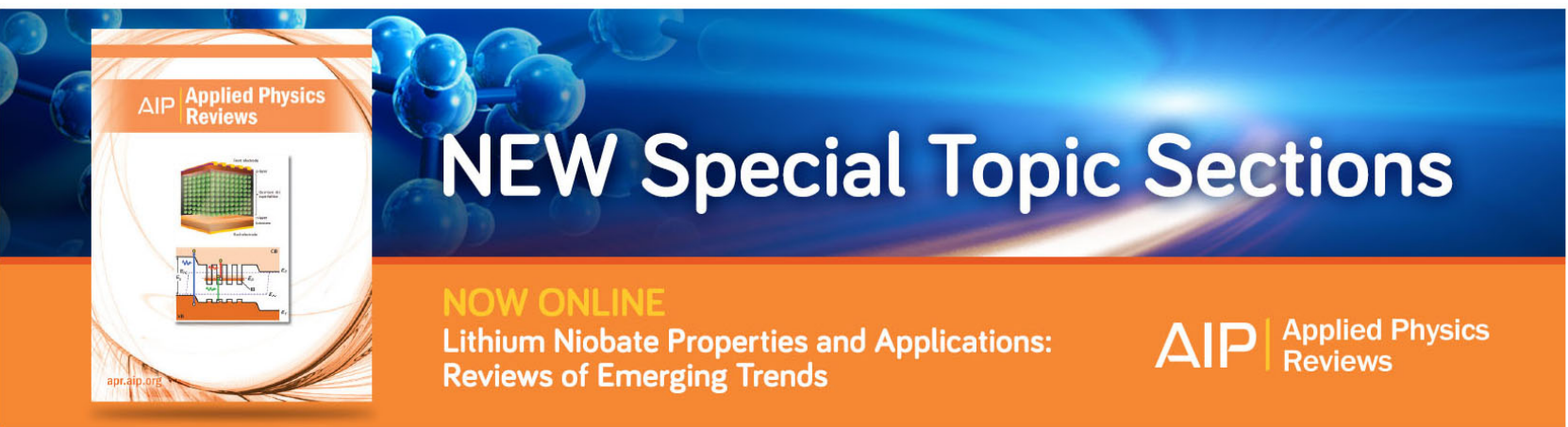




\title{
Mathematical model for self-propelled droplets driven by interfacial tension
}

\author{
Ken H. Nagai, ${ }^{1}$ Kunihito Tachibana, ${ }^{2}$ Yuta Tobe, ${ }^{2}$ Masaki Kazama, ${ }^{2}$ Hiroyuki Kitahata,${ }^{3}$ \\ Seiro Omata, ${ }^{4}$ and Masaharu Nagayama ${ }^{5,6, a)}$ \\ ${ }^{1}$ School of Materials Science, Japan Advanced Institute of Science and Technology, Nomi, \\ Ishikawa 923-1292, Japan \\ ${ }^{2}$ Graduate School of Natural Science and Technology, Kanazawa University, Kanazawa, \\ Ishikawa 920-1192, Japan \\ ${ }^{3}$ Department of Physics, Graduate School of Science, Chiba University, Chiba 263-8522, Japan \\ ${ }^{4}$ Faculty of Mathematics and Physics, Kanazawa University, Kanazawa, Ishikawa 920-1192, Japan \\ ${ }^{5}$ Research Institute for Electronic Science, Hokkaido University, Hokkaido 060-0812, Japan \\ ${ }^{6}$ CREST, Japan Science and Technology Agency, Tokyo 102-0076, Japan
}

(Received 28 December 2015; accepted 26 February 2016; published online 18 March 2016)

\begin{abstract}
We propose a model for the spontaneous motion of a droplet induced by inhomogeneity in interfacial tension. The model is derived from a variation of the Lagrangian of the system and we use a time-discretized Morse flow scheme to perform its numerical simulations. Our model can naturally simulate the dynamics of a single droplet, as well as that of multiple droplets, where the volume of each droplet is conserved. We reproduced the ballistic motion and fission of a droplet, and the collision of two droplets was also examined numerically. (C) 2016 AIP Publishing LLC. [http://dx.doi.org/10.1063/1.4943582]
\end{abstract}

\section{INTRODUCTION}

In far-from-equilibrium systems, it is possible that the symmetry of isotropic systems breaks spontaneously, e.g., spontaneous motion in biological systems. ${ }^{1-3}$ Such selfpropelled phenomena, which are also called "active matter," have recently gained extensive attention, as there is expected to exist general aspects in active matter. Spontaneous motion driven by inhomogeneity in interfacial tension is an area of study relating to active matter. ${ }^{5-16}$ For example, alcohol droplets placed on water move spontaneously while exhibiting deformations to their shape. ${ }^{17,18}$ Since such systems are relatively simple, it is possible to perform experiments under a broad parameter space; therefore, such a setting provides a preferable experimental framework for investigations of general aspects.

One of the authors has constructed a model for the motion of a disk driven by interfacial tension inhomogeneity. ${ }^{19}$ Although it was possible to reproduce and analyze the experimental results for the motion of a camphor disk by use of their model, the same approach is not applicable to the motion of droplets due to the rigid body assumption of the disk. In particular, droplet shape deformations are not negligible, since the coupling between the shape and motion plays an important role in the self-motion of the droplet as has been reported in previous works. ${ }^{17,18,20-25}$ Several studies have already been made for the modeling of a deformable self-propelled droplet. ${ }^{26-33}$ However, in the case of multiple droplets, the individual volumes are not precisely maintained in these models; hence the droplets exchange mass. Since the experimental system we consider here is macroscopic, such volume changes are usually very slow when compared

\footnotetext{
a) Author to whom correspondence should be addressed. Electronic mail: nagayama@es.hokudai.ac.jp.
}

with the time of motion of the droplets; therefore, these models have difficulties in analyzing the dynamics of multiple droplets. What is more, not only the motion of single objects but also the collective dynamics of active matter has recently attracted attention; ${ }^{34-38}$ thus a theoretical model to analyze the dynamics of multiple droplets is needed. In this article, we propose a model, based on a naturally constructed Lagrangian form, for the spontaneous motion of a deformable droplet driven by interfacial tension. Not only the motion of a single droplet but also the fission, fusion, and reflection of multiple droplets can be reproduced using our model. Moreover, the proper volume of each droplet is conserved.

\section{EXPERIMENT}

We first explain the experimental setup which we model. ${ }^{17}$ A schematic diagram of the experimental setup is shown in Fig. 1(a). Two hundred milliliters of aqueous solution of 1-pentanol $(2.2$ vol. \%) was poured into a Petri dish with a diameter of $24 \mathrm{~cm}$, and then a droplet of 1-pentanol was placed on the aqueous phase at $20^{\circ} \mathrm{C}$. To observe the motion of the droplet, the shadowgraph method was used with a digital video camera (iVIS HV30; Canon, Japan). ${ }^{39}$

Figures 1 and 2 show the results of the experiment. A droplet with a volume of $10 \mu \mathrm{l}$ moved ballistically as shown in Fig. 1(b). Alcohol diffuses onto the water surface from the alcohol droplet and the concentration distribution becomes asymmetric due to the deformation of the droplet. Therefore, the interfacial tension of water in front of the droplet becomes higher than that in the rear and the droplet moves unidirectionally.

When the volume of the droplet was increased to $300 \mu \mathrm{l}$, the droplet split into smaller droplets, as shown in Fig. 1(c). Fig. 2 shows the interaction between two droplets that were 
(a)

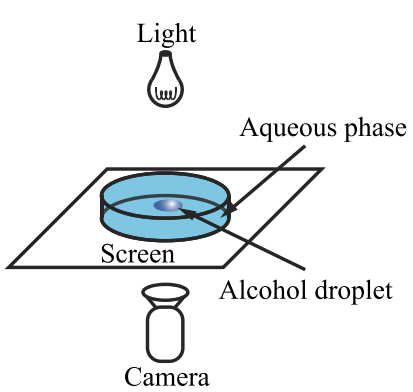

(b)

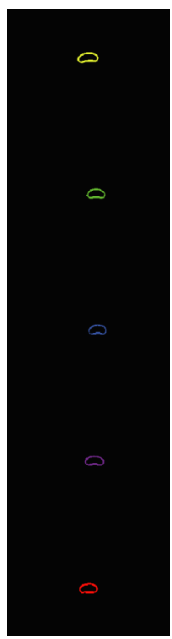

$5 \mathrm{~cm}$ (c)

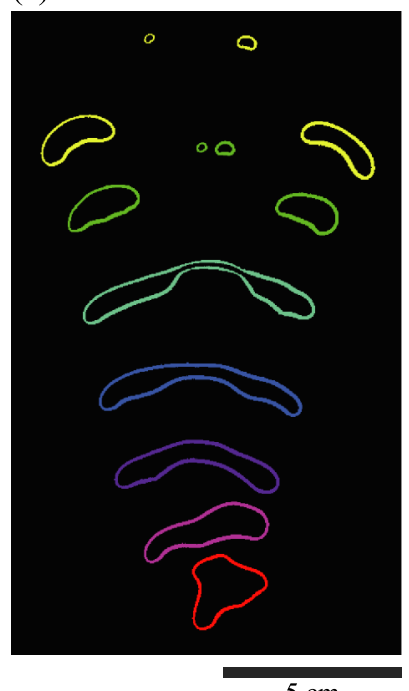

$5 \mathrm{~cm}$

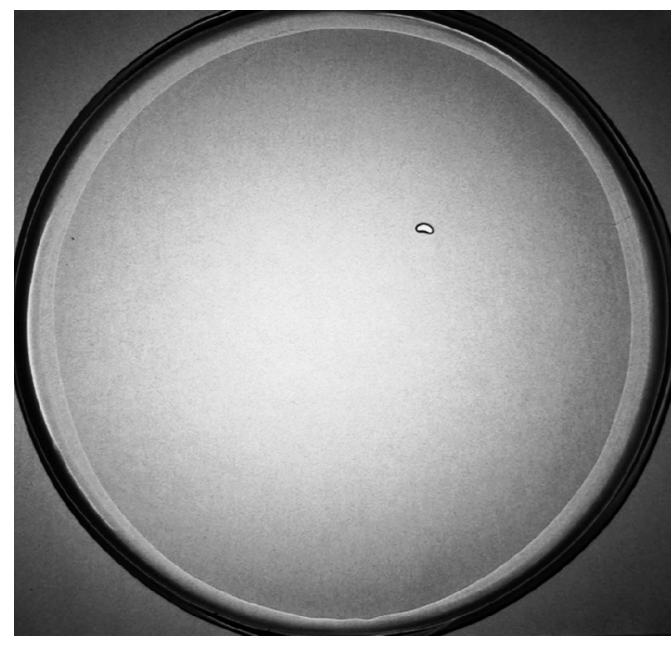

$5 \mathrm{~cm}$

FIG. 1. Experimental setup and characteristic motion of a droplet. (a) Experimental setup for observing the motion of an alcohol droplet on an aqueous phase (1-pentanol solution with a concentration of $2.2 \mathrm{vol}$. \%). The shadowgraph method was used to observe the motion of the droplet. (b) Ballistic motion of the alcohol droplet with a volume of $10 \mu$ l. The outlines of the droplet per $1 \mathrm{~s}$ are shown in the left figure. The droplet moved upward. The right figure is a snapshot of the droplet. (c) Fission of a droplet $(300 \mu 1)$. The outlines of the droplets are shown per $0.67 \mathrm{~s}$. The droplets moved upward. The same color represents the outline at the same time. The droplet elongated before splitting into smaller droplets.

formed as a result of the fission of a single droplet. After the split, droplets of various volumes formed and each continued moving on the water. Here, pairs of droplets were basically found to interact repulsively; when they approached each other at a small angle, the droplets bounced off one another (Fig. 2(a)). In contrast, two droplets fused together when they collided head-on as shown in Fig. 2(b).

\section{MATHEMATICAL MODEL}

To model the dynamics of alcohol droplets, we first constructed a mathematical model for the motion of a single droplet. The schematic illustration of the system is shown

(a)

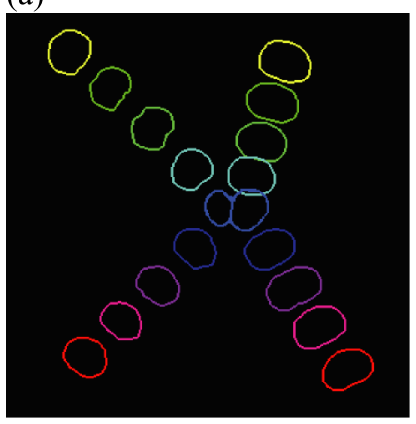

(b)

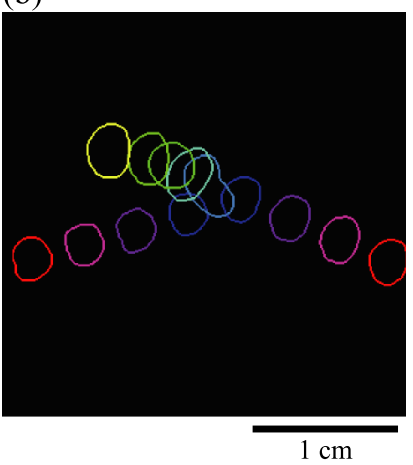

FIG. 2. Collision of two droplets of approximately the same size. The droplets moved upward. The outlines of the droplets are shown per $0.067 \mathrm{~s}$. The same color represents the outline at the same time. (a) Two droplets with a small approach angle bounced off each other. (b) Front-on collision of two droplets. Droplets fused into a single droplet.

in Fig. 3. Although the droplet had a lens-like shape as in Fig. 3(a), the thickness of the droplet is supposed to be so thin that we consider the system in which a droplet is located on a flat water surface as in Fig. 3(b). Actually, the thickness of the droplet is calculated to be around $1 \mathrm{~mm}$ considering the surface tension and density difference. ${ }^{40}$ The hydrodynamic flow may affect the motion of the droplet, but we only consider the effect of the tension gradient at the droplet as a driving force in the present framework for simplicity. In our model, the $x-y$ plane corresponds to the water surface, which we assume is confined to a domain $\Omega$. The height of the droplet from the water surface is $u(t, x, y)$, where $u$ is exactly 0 except for the area under the droplet, and interfacial tensions at the alcohol-air, air-water, and alcohol-water interfaces are $\gamma_{\mathrm{a}}, \gamma_{\mathrm{w}}$, and $\gamma_{\text {wa }}$, respectively. The total interfacial energy in the system is then given by the equation

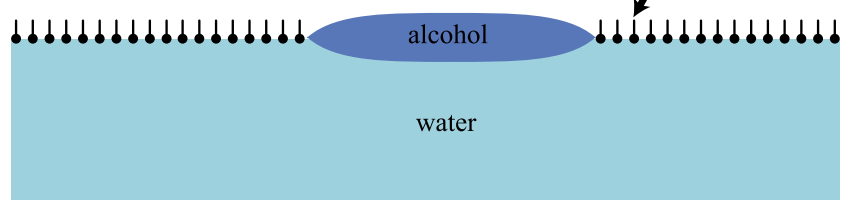

(b)

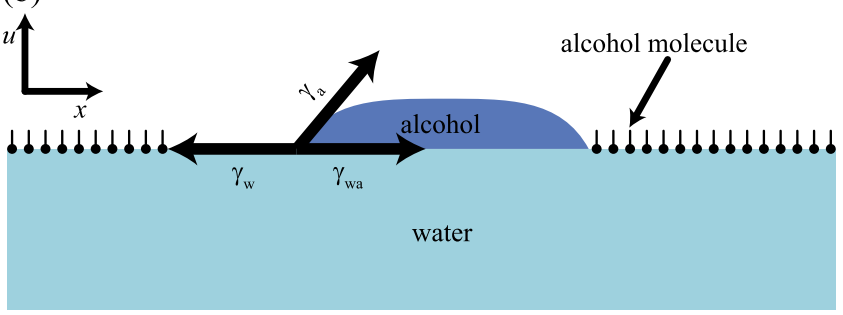

FIG. 3. (a) Schematic illustration of the actual shape of a lens-shaped alcohol droplet. (b) Schematic illustration considered in our model. A droplet is placed on a flat water surface ( $x-y$ plane). The height of the droplet from the $x-y$ plane is $u(x, y)$ and the alcohol concentration under the droplet is $v_{0}$. The interfacial tensions of the alcohol-air, water-air, and alcohol-water interfaces are $\gamma_{\mathrm{a}}, \gamma_{\mathrm{w}}$, and $\gamma_{\mathrm{wa}}$, respectively. The quantity $\gamma_{\mathrm{w}}$ depends on the concentration of alcohol on the water surface, $v(x, y)$. 


$$
\begin{aligned}
U_{1} & =\int_{\Omega}\left(\left(\gamma_{\mathrm{a}} \sqrt{1+|\vec{\nabla} u|^{2}}+\gamma_{\mathrm{wa}}\right) \chi_{u>0}+\gamma_{\mathrm{w}} \chi_{u=0}\right) \mathrm{d} \vec{x} \\
& =\int_{\Omega}\left(\left(\gamma_{\mathrm{a}} \sqrt{1+|\vec{\nabla} u|^{2}}+\gamma_{\mathrm{wa}}\right) \chi_{u>0}+\gamma_{\mathrm{w}}\left(1-\chi_{u>0}\right)\right) \mathrm{d} \vec{x} \\
& =\int_{\Omega}\left(\gamma_{\mathrm{a}} \sqrt{1+|\vec{\nabla} u|^{2}}+\gamma_{\mathrm{wa}}-\gamma_{\mathrm{w}}\right) \chi_{u>0} \mathrm{~d} \vec{x}+\int_{\Omega} \gamma_{\mathrm{w}} \mathrm{d} \vec{x},
\end{aligned}
$$

where $\chi_{u=0}$ and $\chi_{u>0}$ denote the characteristic functions of their set,

$$
\chi_{u=0}= \begin{cases}0 & (u>0), \\ 1 & (u=0)\end{cases}
$$

and

$$
\chi_{u>0}= \begin{cases}1 & (u>0) \\ 0 & (u=0)\end{cases}
$$

We consider thin droplets, where gravity has a large effect. Therefore, the following gravitational energy of a droplet, $U_{2}$, is added to the Lagrangian:

$$
U_{2}=\frac{1}{2} \int_{\Omega} \rho g u^{2} \mathrm{~d} \vec{x},
$$

where $\rho$ is the density difference between alcohol and water, and $g$ is the acceleration of gravity. Assuming $\partial u / \partial t(\vec{x})$ is proportional to the flow speed of the alcohol, the kinetic energy of the system can be expressed as

$$
K=\frac{1}{2} \int_{\Omega} \sigma u\left(\frac{\partial u}{\partial t}\right)^{2} \chi_{u>0} \mathrm{~d} \vec{x},
$$

where $\sigma$ is a proportional coefficient. If the droplet has the large volume under the gravity, we can regard the height of the droplet as fixed constant because the gravitational energy is much larger than the interfacial energy. Therefore, the kinetic energy can be rewritten as

$$
K=\frac{1}{2} \sigma u_{0} \int_{\Omega}\left(\frac{\partial u}{\partial t}\right)^{2} \chi_{u>0} \mathrm{~d} \vec{x},
$$

where $u_{0}$ is the height of the droplet. Assuming $|\nabla u|^{2} \ll 1$, the Lagrangian of the system is described as follows:

$$
\begin{aligned}
L(u)= & K-U_{1}-U_{2} \\
= & \frac{1}{2} \int_{\Omega}\left(-\gamma_{\mathrm{a}}|\vec{\nabla} u|^{2}-2\left(\gamma_{\mathrm{a}}+\gamma_{\mathrm{wa}}-\gamma_{\mathrm{w}}\right)\right. \\
& \left.-\rho g u^{2}+\sigma u_{0}\left(\frac{\partial u}{\partial t}\right)^{2}\right) \chi_{u>0} \mathrm{~d} \vec{x}-\int_{\Omega} \gamma_{\mathrm{w}} \mathrm{d} \vec{x} .
\end{aligned}
$$

The first variation of the action integral corresponding to Eq. (7) under the volume constraint is calculated as

$$
\sigma u_{0} u_{t t}=\gamma_{\mathrm{a}} \Delta u-\rho g u+\lambda,(u>0),
$$

where $\lambda$ is a Lagrange multiplier,

$$
\lambda=\frac{1}{V} \int_{\Omega}\left(\gamma_{\mathrm{a}}|\nabla u|^{2}+\sigma u_{0} u u_{t t} \chi_{u>0}+\rho g u^{2}\right) \mathrm{d} \vec{x} .
$$

At the boundary of the droplet, we also find that $u$ evolves according to

$$
\gamma_{\mathrm{a}}|\vec{\nabla} u|^{2}-\sigma u_{0}\left(u_{t}\right)^{2}=2\left(\gamma_{\mathrm{a}}+\gamma_{\mathrm{wa}}-\gamma_{\mathrm{w}}\right) .
$$

Here, $\vec{\nabla} u=\tan \theta$, where $\theta$ is a contact angle. Since $1-(\tan \theta)^{2} / 2 \approx \cos \theta$, Eq. (10) can be rewritten as

$$
\frac{\sigma}{2}\left(u_{t}\right)^{2}+\gamma_{\mathrm{a}} \cos \theta=\gamma_{\mathrm{w}}-\gamma_{\mathrm{wa}} \text {. }
$$

It is noted that this equation is Young's relation, which is a well-known equation for the contact angle under equilibrium conditions, ${ }^{41}$ when $u_{t}=0$. The derivations of Eqs. (8)-(10) are explained in the Appendix.

We assume that the concentration of alcohol is maintained at saturated concentration, $v_{0}$, under the droplet. Thus, the time evolution for the concentration of alcohol on water, $v$, can be described as

$$
\begin{gathered}
\frac{\partial v}{\partial t}=d_{v} \Delta v-h(v)+g(u, v), \\
h(v)=k_{1} v, \\
g(u, v)=k_{2}\left(v_{0}-v\right) u,
\end{gathered}
$$

where $d_{v}$ is the diffusion constant of alcohol at the water surface, $k_{1}$ is the rate of evaporation of alcohol, and $k_{2}$ is the rate of supply of alcohol. Here, we neglect the solubility of alcohol since the alcohol is almost saturated in water ${ }^{17}$ and the volume of the droplet is kept constant since the evaporation is slow. In our model, $\gamma_{\mathrm{w}}$ depends on $v$ by the relation

$$
\gamma_{\mathrm{w}}(v)=\frac{\gamma_{0}}{1+\beta v^{4}}+\gamma_{1},
$$

where $\gamma_{0}$ and $\gamma_{1}$ are prescribed scalar values. ${ }^{42,43}$ We remark that it is difficult to solve Eqs. (8)-(10) using a conventional method such as finite difference methods, since the term $u_{t t}$ degenerates at $u=0$ and Eq. (9) includes an integral of the unknown function. However, we are able to overcome these difficulties by employing a variational method known as the discrete Morse flow. ${ }^{44,45}$ The discrete Morse flow is explained in the Appendix. We also include a dissipation term in Eq. (8). This term, written as $\alpha u_{t}$, is given to stabilize the oscillatory motions. In this approach, letting $u_{n}$ be the approximation of $u$ at the $n$th time step, $u_{n}$ is defined as the minimizer of the following functional under the volume constraint condition for the droplet:

$$
J_{n}(u)=\frac{1}{2} \int_{\Omega}\left(\sigma u_{0} \frac{\left|u-2 u_{n-1}+u_{n-2}\right|^{2}}{h^{2}} \chi_{u>0}+\alpha \frac{\left|u-u_{n-1}\right|^{2}}{h}+\gamma_{\mathrm{a}}|\vec{\nabla} u|^{2}+\Gamma(v) \chi_{\epsilon}(u)+\rho g u^{2}\right) \mathrm{d} \vec{x},
$$

where

$$
\Gamma=2\left(\gamma_{\mathrm{a}}+\gamma_{\mathrm{wa}}-\gamma_{\mathrm{w}}\right),
$$

and $\chi_{\epsilon}(u)$ is a smooth continuous function on $0<u \leq \epsilon$,

$$
\chi_{\epsilon}(u)= \begin{cases}1 & (u>\epsilon) \\ 0 & (u=0)\end{cases}
$$

It has been proved that $u_{n}$ obtained by minimizing $J_{n}$ under the volume constraint is an approximate solution to Eqs. (8)-(10). Here, we remark that the volume constraint is not complicated in our approach, since the solution with the constraint is proportional to that without it. ${ }^{44,45}$ 
(a)

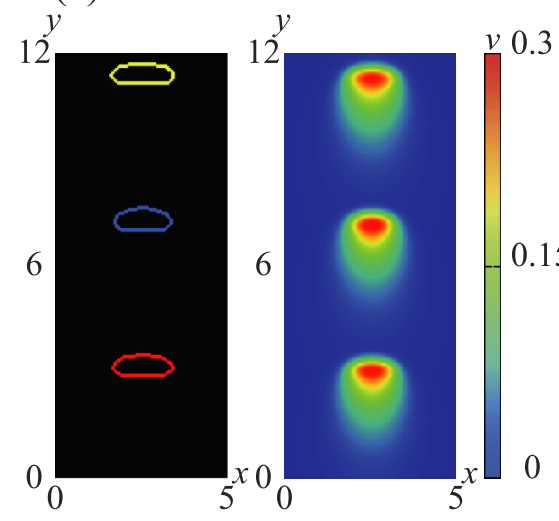

(b)

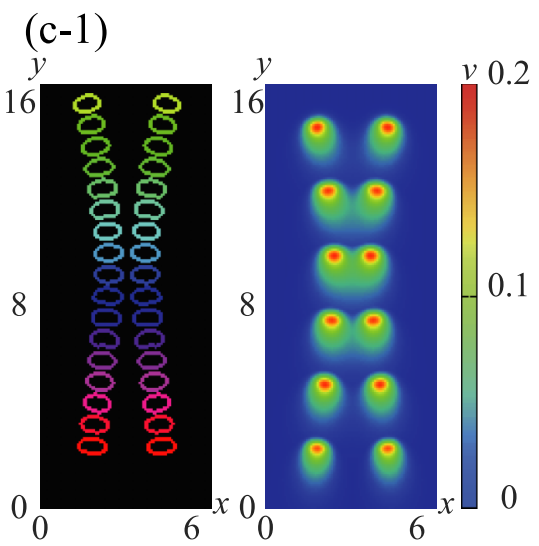

(c-2)

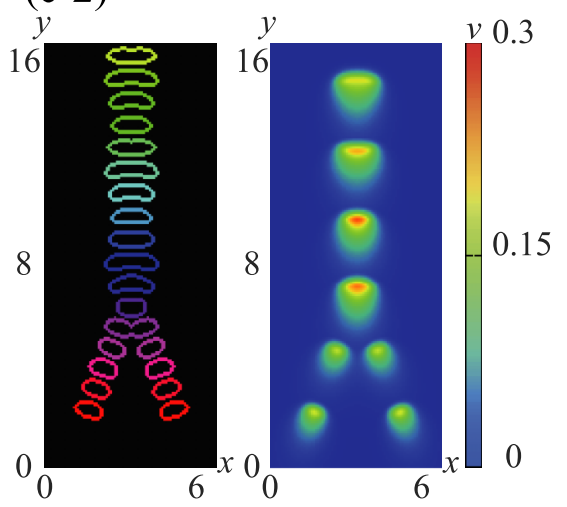

FIG. 4. Numerical results. (a) The motion of a smaller droplet $(V=0.1)$. A droplet deformed into an asymmetric shape and moved ballistically. The outlines of the droplet, which are defined as the closed curves where $u=0.01$ (left), and the snapshots of $v$ with time interval 2.8 (right) are shown. (b) The motion of a larger droplet $(V=2.0)$. The droplet split into smaller droplets. The outlines of the droplets with time interval 1.68 are shown. The same color represents the outline at the same time. (c) Interaction of two droplets $(V=0.035)$ during collision. The outlines of the droplets with time interval 0.7 and the snapshots of $v$ with interval 2.1 are shown. (c-1) Collision with a small approach angle $(7 \pi / 180)$. Droplets bounced off each other. (c-2) Collision with a large approach angle $(\pi / 9)$. Droplets coalesced. The droplet after fusion continued moving upward.

\section{NUMERICAL RESULTS}

The following parameters were used in our numerical simulation, $\epsilon=0.01, \quad h=3.5 \times 10^{-3}, \quad \sigma=2.0, \quad u_{0}=1.0$, $\alpha=2.0, \gamma_{\mathrm{a}}=5.5, \rho=2.0, g=9.8, \gamma_{\mathrm{wa}}=1.56, \gamma_{0}=6.0$, $\gamma_{1}=1.0, \beta=10.0, d_{v}=0.3, k_{1}=1.0, k_{2}=64.0$, and $v_{0}$ $=1.0$. The results of numerical simulation are shown in Fig. 4 . Figure 4(a) shows the motion of a droplet with $V=0.1$. The droplet deformed spontaneously while continuing moving, and the simulations yielded a concentration distribution of alcohol $(v)$ that was higher in the rear of the droplet.

When there are more than one droplet, we can define $J_{n}$ for each droplet since $u$ is identically zero outside the droplet. By minimizing each $J_{n}$, the dynamics of the multiple droplets can be obtained. Redefining the set of $J_{n}$ for each time step, we can simulate the splitting and fusion of the droplets. Actually, when a large droplet $(V=2.0)$ was placed as the initial condition, the droplet elongated and then split into multiple droplets, as shown in Fig. 4(b). This numerical result corresponds well to the experimental result in Fig. 1(b).

We also investigated the interaction between two droplets using this model. Figures 4(c-1) and 4(c-2) show the fusion and repulsion of two droplets, respectively. At the initial state, there were two droplets at the mirror-symmetric positions across the center line. The approach angle, which is defined as the angle of the direction of the droplet motion from the center line, was varied. The direction of motion is calculated as

$$
\frac{\int_{\Pi} \vec{x} \frac{\partial u}{\partial t} \mathrm{~d} \vec{x}}{\left|\int_{\Pi} \vec{x} \frac{\partial u}{\partial t} \mathrm{~d} \vec{x}\right|},
$$

where $\Pi$ is the area including only one droplet. As shown in Fig. 4(c-1), two droplets with small approach angle $(7 \pi / 180)$ reflected and moved apart. On the other hand, when the approach angle was large $(\pi / 9)$, the droplets fused into a single droplet, after which the droplet continued moving upward (Fig. 4(c-2)). It should be noted that the fusion is observed without inertia term ( $\sigma=0$, data not shown).

\section{SUMMARY}

In summary, we proposed a model obtained from variational principles for the motion of alcohol droplets driven by interfacial tension inhomogeneity. Using our model, the dynamics of multiple droplets can be calculated with the volume constraints. We reproduced ballistic motion and fission of a droplet with our model. The interaction of two droplets was also analyzed.

In the present model, we have added the dissipation term. However it is introduced phenomenologically, and the derivation from more fundamental equation such as NavierStokes equation and estimation from the experimental results are remained as future study.

\section{ACKNOWLEDGMENTS}

We thank Professor Kenichi Yoshikawa (Doshisha University, Japan) for his helpful discussion. This work was supported in part by Grants-in-Aid for Exploratory Research to K.H.N. (No. 26610112), for Scientific Research (B) to M.N. (No. 21340023), and Scientific Research (C) to 
H.K. (No. 26520205) from the Ministry of Education, Culture, Sports, Science and Technology of Japan. This work was also supported by the Cooperative Research Program of "Network Joint Research Center for Materials and Devices" to K.H.N. (No. 2015052) and H.K. (Nos. 2014007 and 2015020), and by a Grant for Basic Science Research Projects of The Sumitomo Foundation to K.H.N. (No. 131130).

\section{APPENDIX: DETAILS OF CALCULATIONS}

In this appendix, the derivations of Eqs. (8)-(10) in the main text and the discrete Morse flow are explained.

\section{Derivation of main equations}

First, the derivations of Eqs. (8) and (9) are explained. We set

$$
J(u)=\int_{0}^{\tau} L(u) \mathrm{d} t .
$$

To calculate the first variation of this functional, we perturb using functions from the admissibility set,
$K_{V}:=\left\{u \in W^{1,2}\left(\Omega_{\tau}, \vec{R}\right) ; u=0\right.$ on $\left.\partial \Omega, \int_{\Omega} u \mathrm{~d} \vec{x}=V\right\}$,

where $\Omega_{\tau}=\{(x, t) \mid x \in \Omega, t \in(0, \tau)\}$.

In particular, for any test function $\varphi \in C_{0}^{\infty}\left(\Omega_{\tau} \cap\{u>0\} ; \vec{R}\right)$, we introduce the perturbation $u_{\epsilon}$ as

$$
u_{\epsilon}=V \frac{u+\epsilon \varphi}{V+\epsilon \Phi},
$$

where

$$
\Phi(t)=\int_{\Omega} \varphi(x, y, t) \mathrm{d} \vec{x} .
$$

Then $u_{\epsilon}$ belongs to $K_{V}$,

$$
\begin{gathered}
\int_{\Omega} u_{\epsilon} \mathrm{d} x \mathrm{~d} y=\frac{V}{V+\epsilon \Phi} \int_{\Omega}(u+\epsilon \varphi) \mathrm{d} \vec{x}=V, \\
\left.u_{\epsilon}\right|_{\partial \Omega}=0 .
\end{gathered}
$$

Since $u$ is a stationary point of the functional, the following relation is satisfied:

$$
\lim _{\epsilon \rightarrow 0} \frac{\mathrm{d} J}{\mathrm{~d} \epsilon}\left(u_{\epsilon}\right)=\lim _{\epsilon \rightarrow 0} \frac{J\left(u_{\epsilon}\right)-J(u)}{\epsilon}=0 .
$$

Therefore,

$$
\begin{aligned}
& \lim _{\epsilon \rightarrow 0} \frac{J\left(u_{\epsilon}\right)-J(u)}{\epsilon}=\lim _{\epsilon \rightarrow 0} \frac{\gamma_{\mathrm{a}}}{2 \epsilon} \int_{\Omega_{\tau}}\left[V\left(\frac{|\nabla u+\epsilon \nabla \varphi|}{V+\epsilon \Phi}\right)^{2}-|\nabla u|^{2}\right] \mathrm{d} \vec{z} \\
& -\lim _{\epsilon \rightarrow 0} \frac{\sigma u_{0}}{2 \epsilon} \int_{\Omega_{\tau}}\left[\frac{1}{(V+\epsilon \Phi)^{4}}\left\{\left(u_{t}+\epsilon \varphi_{t}\right)(V+\epsilon \Phi)-(u+\epsilon \varphi) \epsilon \Phi_{t}\right\}^{2} \chi_{u_{\epsilon}>0}-u_{t}^{2} \chi_{u>0}\right] \mathrm{d} \vec{z} \\
& -\lim _{\epsilon \rightarrow 0} \frac{1}{2 \epsilon} \int_{\Omega_{\tau}}\left(R(v) \chi_{u_{\epsilon}>0}-R(v) \chi_{u>0}\right) \mathrm{d} \vec{z}+\lim _{\epsilon \rightarrow 0} \frac{\rho}{2 \epsilon} \int_{\Omega_{\tau}} \frac{g(u+\epsilon \varphi)^{2} V^{2}-g u^{2}(V+\epsilon \Phi)^{2}}{(V+\epsilon \Phi)^{2}} \mathrm{~d} \vec{z} \\
& =\lim _{\epsilon \rightarrow 0} \frac{\gamma_{\mathrm{a}}}{2 \epsilon} \int_{\Omega_{\tau}} \frac{1}{(V+\epsilon \Phi)^{2}}\left\{V^{2}\left(|\nabla u|^{2}+2 \epsilon \nabla u \nabla \varphi+\epsilon^{2}|\nabla \varphi|^{2}\right)-\left(V^{2}+2 \epsilon V \Phi+\epsilon^{2} \Phi^{2}\right)|\nabla u|^{2}\right\} \mathrm{d} \vec{z} \\
& -\lim _{\epsilon \rightarrow 0} \frac{\sigma u_{0}}{2 \epsilon} \int_{\Omega_{\tau}} \frac{1}{(V+\epsilon \Phi)^{4}}\left\{V^{2}\left\{\left(u_{t}+\epsilon \varphi_{t}\right)+\epsilon \Phi V u_{t}-\epsilon \Phi{ }_{t} u\right\}^{2} \chi_{u_{\epsilon}>0}-\left(u_{t}\right)^{2}(V+\epsilon \Phi)^{4} \chi_{u>0}+o(\epsilon)\right\} \mathrm{d} \vec{z} \\
& +\lim _{\epsilon \rightarrow 0} \frac{\rho}{2 \epsilon} \int_{\Omega_{\tau}} \frac{1}{(V+\epsilon \Phi)^{2}}\left\{V^{2}\left(2 g u \epsilon \varphi+g \epsilon^{2} \varphi^{2}\right)-2 g u^{2} \epsilon V \Phi-g u^{2} \epsilon^{2} \Phi^{2}\right\} \mathrm{d} \vec{z} \\
& =\lim _{\epsilon \rightarrow 0} \frac{\gamma_{\mathrm{a}}}{2 \epsilon} \int_{\Omega_{\tau}} \frac{2 V^{2} \epsilon \nabla u \nabla \varphi+2 \epsilon V \Phi|\nabla u|^{2}+o(\epsilon)}{(V+\epsilon \Phi)^{2}} \mathrm{~d} \vec{z} \\
& -\lim _{\epsilon \rightarrow 0} \frac{\sigma u_{0}}{2 \epsilon} \int_{\Omega_{\tau}} \frac{1}{(V+\epsilon \Phi)^{4}}\left\{\left(2 \epsilon u_{t} \varphi_{t} V^{4}+2 \epsilon V^{3} \Phi\left(u_{t}\right)^{2}-2 \epsilon V^{3} \Phi_{t} u u_{t}-4 \epsilon V^{3} \Phi\left(u_{t}\right)^{2}\right) \chi_{u>0}+o(\epsilon)\right\} \mathrm{d} \vec{z} \\
& +\lim _{\epsilon \rightarrow 0} \frac{\rho}{2 \epsilon} \int_{\Omega_{\tau}} \frac{2 g u \epsilon V^{2} \varphi-2 g u^{2} \epsilon V \Phi+o(\epsilon)}{(V+\epsilon \Phi)^{2}} \mathrm{~d} \vec{z} \\
& =\int_{\Omega_{\tau}}\left[\gamma_{\mathrm{a}}\left(\nabla u \nabla \varphi-\frac{1}{V} \Phi|\nabla u|^{2}\right)-\sigma u_{0}\left(u_{t} \varphi_{t}-\frac{1}{V} u_{t}(u \Phi)_{t}\right) \chi_{u>0}+\rho\left(g u \varphi-\frac{1}{V} g u^{2} \Phi\right)\right] \mathrm{d} \vec{z} \\
& =\int_{\Omega_{\tau} \cap\{u>0\}}\left(\gamma_{\mathrm{a}} \nabla u \nabla \varphi-\sigma u_{0} u_{t} \varphi_{t}+\rho g u \varphi\right) \mathrm{d} \vec{z}+\frac{1}{V} \int_{\Omega_{\tau}}\left(-\gamma_{\mathrm{a}}|\nabla u|^{2} \Phi+\sigma u_{0} u_{t}(u \Phi)_{t} \chi_{u>0}-\rho g u^{2} \Phi\right) \mathrm{d} \vec{z} \\
& =0 \text {, }
\end{aligned}
$$


where $\vec{z}=(x, y, t)$. Formally integrating by parts in time and recalling the expression for the Lagrange multiplier, one has

$$
\begin{aligned}
\frac{1}{V} \int_{\Omega_{\tau}}\left(-\gamma_{\mathrm{a}}|\nabla u|^{2} \Phi+\sigma u_{0} u_{t}(u \Phi)_{t} \chi_{u>0}-\rho g u^{2} \Phi\right) \mathrm{d} \vec{z} \\
=\frac{1}{V} \int_{\Omega_{\tau}}\left(-\gamma_{\mathrm{a}}|\nabla u|^{2}-\sigma u_{0} u_{t t} u \chi_{u>0}-\rho g u^{2}\right) \Phi \mathrm{d} \vec{z} \\
=\int_{0}^{\tau}\left(\int_{\Omega} \varphi \mathrm{d} \vec{x}\right)\left(\frac { 1 } { V } \int _ { \Omega } \left(-\gamma_{a}|\nabla u|^{2}-\sigma u_{0} u_{t t} u \chi_{u>0}\right.\right. \\
\left.\left.\quad-\rho g u^{2}\right) \mathrm{~d} \vec{x}\right) \mathrm{d} t \\
=\int_{0}^{\tau}\left(\int_{\Omega} \varphi \mathrm{d} \vec{x}\right)(-\lambda) \mathrm{d} t \\
=-\int_{\Omega_{\tau} \cap\{u>0\}}^{\lambda \varphi \mathrm{d} \vec{z}}
\end{aligned}
$$

where

$$
\lambda=\frac{1}{V} \int_{\Omega}\left(\gamma_{a}|\nabla u|^{2}+\sigma u_{0} u_{t} u \chi_{u>0}+\rho g u^{2}\right) \mathrm{d} \vec{x} .
$$

Therefore, we obtain the following weak formulation for Eq. (8) by use of Lagrange multiplier $\lambda$ :

$$
\begin{gathered}
\int_{\Omega_{\tau} \cap\{u>0\}}\left(\gamma_{a} \nabla u \nabla \varphi-\sigma u_{0} u_{t} \varphi_{t}+\rho g u \varphi-\lambda \varphi\right) \mathrm{d} \vec{z}=0, \\
\forall \varphi \in C_{0}^{\infty}\left(\Omega_{\tau} \cap\{u>0\} ; \vec{R}\right) .
\end{gathered}
$$

By

$$
\int_{\Omega_{\tau} \cap\{u>0\}} \gamma_{a} \nabla u \nabla \varphi \vec{z}=-\int_{\Omega_{\tau} \cap\{u>0\}} \gamma_{a} \nabla^{2} u \varphi \vec{z},
$$

and

$$
\int_{\Omega_{\tau} \cap\{u>0\}} \sigma u_{0} u_{t} \varphi_{t} \vec{z}=-\int_{\Omega_{\tau} \cap\{u>0\}} \sigma u_{0} u_{t t} \varphi \vec{z},
$$

finally we obtain the following formulation:

$$
\begin{gathered}
\int_{\Omega_{\tau} \cap\{u>0\}}\left(-\gamma_{a} \nabla^{2} u+\sigma u_{0} u_{t t}+\rho g u-\lambda\right) \varphi \mathrm{d} \vec{z}=0, \\
\forall \varphi \in C_{0}^{\infty}\left(\Omega_{\tau} \cap\{u>0\} ; \vec{R}\right) .
\end{gathered}
$$

Therefore, Eq. (8) is derived.

\section{Derivation of equation on boundary}

Here, the derivation of Eq. (10) is explained. For simplicity, we set $\gamma_{\mathrm{a}}=\sigma=\rho=u_{0}=1$. For any $\eta \in C_{0}^{\infty}\left(\Omega_{\tau} ; \vec{R}^{3}\right)$, the map,

$$
\begin{gathered}
\tilde{\vec{z}}=\tau_{\varepsilon}(\vec{z}):=\vec{z}+\varepsilon \vec{\eta}(\vec{z}), \\
\tau_{\varepsilon}: \Omega \times(0, \tau) \rightarrow \Omega \times(0, \tau),
\end{gathered}
$$

is used to define a volume-preserving domain perturbation,

$$
u_{\varepsilon}=\frac{V}{V_{\varepsilon}} u\left(\tau_{\varepsilon}^{-1}(\tilde{\vec{z}})\right) .
$$

In this case, the Jacobian of $\tau_{\varepsilon}$ is

$$
\left|D \tau_{\varepsilon}\right|=1+\varepsilon \operatorname{div} \vec{\eta}+o(\varepsilon), \quad(\text { as } \varepsilon \rightarrow 0)
$$

and we write

$$
V_{\varepsilon}=\int_{\Omega} u\left(\tau_{\varepsilon}^{-1}(\tilde{\vec{z}})\right) \mathrm{d} \tilde{\vec{z}}
$$

Then

$$
\begin{aligned}
V_{\varepsilon} & =\int_{\Omega} u\left(\tau_{\varepsilon}^{-1}(\tilde{\vec{z}})\right) \mathrm{d} \tilde{\vec{z}} \\
& =\int_{\Omega} u(\vec{z})\left|D \tau_{\varepsilon}(\vec{z})\right| \mathrm{d} \vec{z} \\
& =V+\varepsilon \int_{\Omega} u(\vec{z}) \operatorname{div} \vec{\eta}(\vec{z}) \mathrm{d} \vec{z}+o(\varepsilon) .
\end{aligned}
$$

Therefore,

$$
\lim _{\varepsilon \downarrow 0} \int_{\Omega} u_{\varepsilon} \mathrm{d} \vec{z}=V,
$$

and the perturbation has the volume constraint property. Next, we record the following relation:

$$
\begin{aligned}
\frac{\partial u_{\varepsilon}}{\partial z_{i}}(\vec{z})= & \frac{V}{V_{\varepsilon}} \sum_{j=1}^{3} \frac{\partial u}{\partial z_{j}}\left(\tau_{\varepsilon}^{-1}(\tilde{\vec{z}})\right) \frac{\partial\left(\tau_{\varepsilon}^{-1}\right)_{j}}{\partial z_{i}}(\tilde{\vec{z}}) \\
= & \frac{V}{V_{\varepsilon}} \sum_{j=1}^{3} \frac{\partial u}{\partial z_{j}}\left(\tau_{\varepsilon}^{-1}(\tilde{\vec{z}})\right)\left(D \tau_{\varepsilon}\right)_{j i}^{-1}\left(\tau_{\varepsilon}^{-1}(\tilde{\vec{z}})\right) \\
= & \frac{V}{V_{\varepsilon}} \sum_{j=1}^{3} \frac{\partial u}{\partial z_{j}}\left(\tau_{\varepsilon}^{-1}(\tilde{\vec{z}})\right) \\
& \times\left(\delta_{i j}-\varepsilon \frac{\partial \eta_{j}}{\partial z_{i}}\left(\tau_{\varepsilon}^{-1}(\tilde{\vec{z}})\right)+o(\varepsilon)\right),
\end{aligned}
$$

where $\delta_{i j}$ denotes the Kronecker delta function. Calculating the inner variation, one has

$$
\begin{aligned}
\lim _{\varepsilon \rightarrow 0} \frac{1}{\varepsilon}\left(J\left(u_{\varepsilon}\right)-J(u)\right)= & \lim _{\varepsilon \rightarrow 0} \frac{1}{2 \varepsilon} \int_{\Omega_{\tau}}\left\{\frac{V^{2}}{V_{\varepsilon}^{2}} \sum_{i=1}^{2}\left(\frac{\partial u}{\partial z_{i}}-\varepsilon \sum_{j=1}^{3} \frac{\partial u}{\partial z_{j}} \frac{\partial \eta_{j}}{\partial z_{i}}\right)^{2}(1+\varepsilon \operatorname{div} \vec{\eta})-|\nabla u|^{2}\right\} \mathrm{d} \tilde{\vec{z}} \\
& +\lim _{\varepsilon \rightarrow 0} \frac{1}{2 \varepsilon} \int_{\Omega_{\tau}}\left(R\left(v\left(\tau_{\varepsilon}\right)\right)(1+\varepsilon \operatorname{div} \vec{\eta})-R(v)\right) \chi_{u>0} \mathrm{~d} \tilde{\vec{z}}+\lim _{\varepsilon \rightarrow 0} \frac{1}{2 \varepsilon} \int_{\Omega_{\tau}}\left\{\frac{V^{2}}{V_{\varepsilon}^{2}} g u^{2}(1+\varepsilon \operatorname{div} \vec{\eta})-g u^{2}\right\} \mathrm{d} \tilde{\vec{z}} \\
& -\lim _{\varepsilon \rightarrow 0} \frac{1}{2 \varepsilon} \int_{\Omega_{\tau}}\left\{\frac{V^{2}}{V_{\varepsilon}^{2}}\left(\frac{\partial u}{\partial t}-\varepsilon \sum_{j=1}^{3} \frac{\partial u}{\partial z_{j}} \frac{\partial \eta_{j}}{\partial t}\right)^{2}-u_{t}^{2}\right\} \chi_{u>0} \mathrm{~d} \tilde{\vec{z}} \\
& +\lim _{\varepsilon \rightarrow 0} \frac{1}{\varepsilon} \int_{\Omega_{\tau}}\left\{\gamma_{\mathrm{wa}}\left(v\left(\tau_{\varepsilon}\right)\right)(1+\varepsilon \operatorname{div} \vec{\eta})-\gamma_{\mathrm{wa}}(v)\right\} \mathrm{d} \tilde{\vec{z}} \\
= & 0,
\end{aligned}
$$




$$
\begin{aligned}
& \lim _{\varepsilon \rightarrow 0} \frac{1}{\varepsilon} \int_{\Omega_{\tau}} \frac{1}{V^{2}+2 \varepsilon V \int_{\Omega} u \operatorname{div} \vec{\eta} \mathrm{d} \vec{x}+o(\varepsilon)} \\
& \left\{V^{2} \sum_{i=1}^{2}\left(\frac{\partial u}{\partial z_{i}}-\varepsilon \sum_{j=1}^{3} \frac{\partial u}{\partial z_{j}} \frac{\partial \eta_{j}}{\partial z_{i}}\right)^{2}(1+\varepsilon \operatorname{div} \vec{\eta})-|\nabla u|^{2}\left(V^{2}+2 \varepsilon V \int_{\Omega} u \operatorname{div} \vec{\eta} \mathrm{d} \vec{x}+o(\varepsilon)\right)\right\} \mathrm{d} \tilde{\vec{z}} \\
& +\lim _{\varepsilon \rightarrow 0} \frac{1}{2} \int_{\Omega_{\tau}}\left\{\frac{R\left(v\left(\tau_{\varepsilon}\right)\right)-R(v)}{\varepsilon}+R\left(v\left(\tau_{\varepsilon}\right)\right) \operatorname{div} \vec{\eta}\right\} \chi u>0 \mathrm{~d} \tilde{\vec{z}}+\lim _{\varepsilon \rightarrow 0} \frac{1}{2 \varepsilon} \int_{\Omega_{\tau}} \frac{1}{V^{2}+2 \varepsilon V \int_{\Omega} u \operatorname{div} \vec{\eta} \mathrm{d} \vec{x}+o(\varepsilon)} \\
& \left\{V^{2} g u^{2}(1+\varepsilon \operatorname{div} \vec{\eta})-g u^{2}\left(V^{2}+2 \varepsilon V \int_{\Omega} u \operatorname{div} \vec{\eta} \mathrm{d} \vec{x}+o(\varepsilon)\right)\right\} \mathrm{d} \tilde{\vec{z}} \\
& -\lim _{\varepsilon \rightarrow 0} \frac{1}{2 \varepsilon} \int_{\Omega_{\tau}} \frac{1}{V^{2}+2 \varepsilon V \int_{\Omega} u \operatorname{div} \vec{\eta} \mathrm{d} \vec{x}+o(\varepsilon)} \\
& \left\{V^{2}\left(\frac{\partial u}{\partial t}-\varepsilon \sum_{j=1}^{3} \frac{\partial u}{\partial z_{j}} \frac{\partial \eta_{j}}{\partial t}\right)^{2}(1+\varepsilon \operatorname{div} \vec{\eta})-u_{t}^{2}\left(V^{2}+2 \varepsilon V \int_{\Omega} u \operatorname{div} \vec{\eta} \mathrm{d} \vec{x}+o(\varepsilon)\right)\right\} \chi_{u>0} \mathrm{~d} \tilde{\vec{z}} \\
& +\lim _{\varepsilon \rightarrow 0} \int_{\Omega_{\tau}}\left(\frac{\gamma_{\mathrm{wa}}\left(v\left(\tau_{\varepsilon}\right)\right)-\gamma_{\mathrm{wa}}(v)}{\varepsilon}+\gamma_{\mathrm{wa}}\left(v\left(\tau_{\varepsilon}\right)\right) \operatorname{div} \vec{\eta}\right) \mathrm{d} \tilde{\vec{z}} \\
& =0 \text {, } \\
& \int_{\Omega_{\tau} \cap\{u>0\}}\left\{-\sum_{i=1}^{2} \sum_{j=1}^{3} \frac{\partial u}{\partial z_{i}} \frac{\partial u}{\partial z_{j}} \frac{\partial \eta_{j}}{\partial z_{i}}+\frac{1}{2}|\nabla u|^{2} \operatorname{div} \vec{\eta}-\frac{|\nabla u|^{2}}{V} \int_{\Omega} u \operatorname{div} \vec{\eta} \mathrm{d} \vec{x}\right\} \mathrm{d} \tilde{\vec{z}} \\
& +\frac{1}{2} \int_{\Omega_{\tau} \cap\{u>0\}} \operatorname{div}(R(v) \vec{\eta}) \mathrm{d} \tilde{\vec{z}}+\int_{\Omega_{\tau} \cap\{u>0\}}\left(\frac{g u^{2}}{2} \operatorname{div} \vec{\eta}-\frac{g u^{2}}{V} \int_{\Omega} u \operatorname{div} \vec{\eta} \mathrm{d} \vec{x}\right) \mathrm{d} \vec{z} \\
& -\int_{\Omega_{\tau} \cap\{u>0\}}\left\{-\sum_{j=1}^{3} \frac{\partial u}{\partial t} \frac{\partial u}{\partial z_{j}} \frac{\partial \eta_{j}}{\partial t}+\frac{u_{t}^{2}}{2} \operatorname{div} \vec{\eta}-\frac{u_{t}^{2} \chi_{u>0}}{V} \int_{\Omega} u \operatorname{div} \vec{\eta} \mathrm{d} x\right\} \mathrm{d} \vec{z}+\int_{\Omega_{\tau}} \operatorname{div}\left(\gamma_{\mathrm{wa}}(v) \vec{\eta}\right) \mathrm{d} \vec{z} \\
& =0 \text {. }
\end{aligned}
$$

Using the expression for the Lagrange multiplier, one has

$$
\begin{aligned}
\int_{\Omega_{\tau} \cap\{u>0\}} & \left\{-\sum_{i=1}^{3} \sum_{j=1}^{3} \frac{\partial u}{\partial z_{i}} \frac{\partial u}{\partial z_{j}} \frac{\partial \eta_{j}}{\partial z_{i}}+\frac{1}{2}|\nabla u|^{2} \operatorname{div} \vec{\eta}\right. \\
& +\frac{1}{2} \operatorname{div}(R(v) \vec{\eta})+\frac{g u^{2}}{2} \operatorname{div} \vec{\eta} \\
& \left.+\sum_{j=1}^{3} \frac{\partial u}{\partial t} \frac{\partial u}{\partial z_{j}} \frac{\partial \eta_{j}}{\partial t}-\frac{u_{t}^{2}}{2} \operatorname{div} \vec{\eta}-\lambda u \operatorname{div} \vec{\eta}\right\} \mathrm{d} \tilde{\vec{z}} \\
& +\int_{\Omega_{\tau}} \operatorname{div}\left(\gamma_{\mathrm{wa}}(v) \vec{\eta}\right) \mathrm{d} \tilde{\vec{z}}=0,
\end{aligned}
$$

which, upon defining

$$
\begin{aligned}
\nabla_{h} u & =\left(\frac{\partial u}{\partial x_{1}}, \frac{\partial u}{\partial x_{2}},-\frac{\partial u}{\partial t}\right) \\
\nabla_{z} u & =\left(\frac{\partial u}{\partial x_{1}}, \frac{\partial u}{\partial x_{2}}, \frac{\partial u}{\partial t}\right)
\end{aligned}
$$

can be written as

$$
\begin{aligned}
\int_{\Omega_{\tau} \cap\{u>0\}} & \left\{\frac{1}{2}\left(\nabla_{z} \cdot \vec{\eta}\right)\left(\nabla_{z} u \cdot \nabla_{h} u\right)-\nabla_{z} u D \vec{\eta} \nabla_{h} u\right. \\
& \left.+\frac{1}{2} \operatorname{div}(R(v) \vec{\eta})+\frac{g u^{2}}{2} \operatorname{div} \vec{\eta}-\lambda u \operatorname{div} \vec{\eta}\right\} \mathrm{d} \tilde{\vec{z}} \\
& +\int_{\Omega_{\tau}} \operatorname{div}\left(\gamma_{\text {wa }}(v) \vec{\eta}\right) \mathrm{d} \tilde{\vec{z}} \\
= & 0 .
\end{aligned}
$$

We also note the following relation:

$$
\begin{aligned}
\frac{1}{2}\left(\nabla_{z} \cdot \vec{\eta}\right) & \left(\nabla_{z} u \cdot \nabla_{h} u\right)-\nabla_{z} u D \vec{\eta} \nabla_{h} u \\
& -\left(\vec{\eta} \cdot \nabla_{z} u\right)\left(\Delta u-u_{t t}-g u+\lambda\right)+\frac{1}{2} \operatorname{div}(R(v) \vec{\eta}) \\
& +\frac{g u^{2}}{2} \operatorname{div} \vec{\eta}-\lambda u \operatorname{div} \vec{\eta} \\
= & \nabla_{z} \cdot\left[\frac{1}{2} \vec{\eta}\left(\nabla_{z} u \cdot \nabla_{h} u\right)-\left(\vec{\eta} \cdot \nabla_{z} u\right) \nabla_{h} u+\frac{1}{2} R(v) \vec{\eta}\right. \\
& \left.+\frac{g u^{2}}{2} \vec{\eta}-\lambda u \vec{\eta}\right],
\end{aligned}
$$

so that, upon using Green's theorem, we obtain

$$
\begin{aligned}
\int_{\Omega_{\tau} \cap\{u>0\}} & {\left[\nabla _ { z } \cdot \left[\frac{1}{2} \vec{\eta}\left(\nabla_{z} u \cdot \nabla_{h} u\right)-\left(\vec{\eta} \cdot \nabla_{z} u\right) \nabla_{h} u\right.\right.} \\
& \left.\left.+\frac{1}{2} R(v) \vec{\eta}+\frac{g u^{2}}{2} \vec{\eta}+\lambda u \vec{\eta}\right]\right] \mathrm{d} \tilde{\vec{z}} \\
& +\int_{\Omega_{\tau}} \operatorname{div}\left(\gamma_{\mathrm{wa}}(v) \vec{\eta}\right) \mathrm{d} \tilde{\vec{z}} \\
= & \frac{1}{2} \int_{\Omega_{\tau} \cap \partial\{u>0\}}\left[\left(\nabla_{z} u \cdot \nabla_{h} u\right)(\vec{\eta} \cdot \vec{v})\right. \\
& \left.-2\left(\vec{\eta} \cdot \nabla_{z} u\right)\left(\nabla_{h} u \cdot \vec{v}\right)+R(v)(\vec{\eta} \cdot \vec{v})\right] \mathrm{d} S \\
= & 0,
\end{aligned}
$$


where $\vec{v}$ is the outer normal vector on $\Omega_{\tau} \cap\{u>0\}$. Since $\vec{v}=-\nabla_{z} u /\left|\nabla_{z} u\right|$, one has

$$
\begin{gathered}
\int_{\Omega_{\tau} \cap \partial\{u>0\}}\left(-|\nabla u|^{2}+u_{t}^{2}+R(v)\right)(\vec{v} \cdot \vec{\eta}) \mathrm{d} S=0, \\
\forall \vec{\eta} \in C_{0}^{\infty}\left(\Omega_{\tau} ; \vec{R}^{3}\right),
\end{gathered}
$$

and hence the following condition holds on the free boundary:

$$
|\nabla u|^{2}-\left(u_{t}\right)^{2}=R(v) \quad \text { on } \Omega_{\tau} \cap \partial\{u>0\} .
$$

\section{Discrete Morse flow}

The discrete Morse flow builds a sequence of weak solutions to approximating elliptic problems by minimizing $J_{n}(u)$ under the volume constraint condition and obtains a solution to original problems (8)-(10) by taking the discretized parameter $h$ to zero. ${ }^{46}$

For example, considering the functional,

$H_{n}(u)=\int_{\Omega}\left(\frac{\left|u-2 u_{n-1}+u_{n-2}\right|^{2}}{2 h^{2}} \chi_{u>0}+\frac{|\nabla u|^{2}}{2}\right) \mathrm{d} x$,

and using perturbations $u_{\epsilon}=u+\epsilon \phi(x)$ where $\phi \in C_{0}^{\infty}(u>0)$, we compute the Euler-Lagrange equation,

$$
\begin{gathered}
\left.\frac{d}{d \epsilon} H_{n}\left(u_{\epsilon}\right)\right|_{\epsilon=0}=0 \\
\int_{\Omega}\left(\frac{u-2 u_{n-1}+u_{n-2}}{h^{2}} \phi+\nabla u \nabla \phi\right) \mathrm{d} x=0 .
\end{gathered}
$$

Formally taking $h \rightarrow 0$ in the above equation yields

$$
\int_{\Omega}\left(u_{t t} \phi+\nabla u \nabla \phi\right) \mathrm{d} x=0, \quad\left(\forall \phi \in C_{0}^{\infty}(u>0)\right),
$$

which is a weak formulation for the wave equation (inside the free boundary),

$$
u_{t t}=\Delta u \quad \text { in }\{u>0\} .
$$

Therefore, by successively minimizing $H_{n}(u)$, we can construct a sequence of functions approximating the weak solution to the above wave equation.

${ }^{1}$ F. Schweitzer, W. Ebeling, and B. Tilch, Phys. Rev. Lett. 80, 5044 (1998).

${ }^{2}$ A. B. Verkhovsky, T. M. Svitkina, and G. G. Borisy, Curr. Biol. 9, 11 (1999).

${ }^{3}$ R. Golestanian, T. B. Liverpool, and A. Ajdari, Phys. Rev. Lett. 94, 220801 (2005).

${ }^{4}$ S. Ramaswamy, Annu. Rev. Condens. Matter Phys. 1, 323 (2010).

${ }^{5}$ Lord Rayleigh, Proc. R. Soc. London 47, 364 (1890).

${ }^{6}$ C. D. Bain, G. D. Burnett-Hall, and R. R. Montgomerie, Nature 372, 414 (1994).

${ }^{7}$ F. D. Dos Santos and T. Ondarçuhu, Phys. Rev. Lett. 75, 2972 (1995).
${ }^{8}$ S. Nakata, Y. Iguchi, S. Ose, M. Kuboyama, T. Ishii, and K. Yoshikawa, Langmuir 13, 4454 (1997).

${ }^{9}$ H. Kitahata, R. Aihara, N. Magome, and K. Yoshikawa, J. Chem. Phys. 116, 5666 (2002).

${ }^{10}$ S. Lee, D. Y. Kwok, and P. E. Laibinis, Phys. Rev. E 65, 051602 (2002).

${ }^{11}$ Y. Sumino, M. Nagayama, H. Kitahata, S. M. Nomura, N. Magome, Y. Mori, and K. Yoshikawa, Phys. Rev. E 72, 041603 (2005).

${ }^{12}$ T. Toyota, N. Maru, M. M. Hanczyc, T. Ikegami, and T. Sugawara, J. Am. Chem. Soc. 131, 5012 (2009).

${ }^{13}$ H. Kitahata, N. Yoshinaga, K. H. Nagai, and Y. Sumino, Phys. Rev. E 84, 015101(R) (2011).

${ }^{14}$ F. Takabatake, N. Magome, M. Ichikawa, and K. Yoshikawa, J. Chem. Phys. 134, 114704 (2011).

${ }^{15}$ S. Yabunaka, T. Ohta, and N. Yoshinaga, J. Chem. Phys. 136, 074904 (2012).

${ }^{16} \mathrm{~F}$. Takabatake, K. Yoshikawa, and M. Ichikawa, J. Chem. Phys. 141, 051103 (2014).

${ }^{17}$ K. Nagai, Y. Sumino, H. Kitahata, and K. Yoshikawa, Phys. Rev. E 71, 065301(R) (2005)

${ }^{18}$ K. Nagai, Y. Sumino, and K. Yoshikawa, Colloids Surf., B 56, 197 (2007).

${ }^{19}$ M. Nagayama, S. Nakata, Y. Doi, and Y. Hayashima, Physica D 194, 151 (2004).

${ }^{20}$ K. Krischer and A. Mikhailov, Phys. Rev. Lett. 73, 3165 (1994).

${ }^{21}$ T. Ohta and T. Ohkuma, Phys. Rev. Lett. 102, 154101 (2009).

${ }^{22}$ Y. Itino, T. Ohkuma, and T. Ohta, J. Phys. Soc. Jpn. 80, 033001 (2011)

${ }^{23}$ Y. Itino and T. Ohta, J. Phys. Soc. Jpn. 81, 104007 (2012).

${ }^{24}$ H. Kitahata, K. Iida, and M. Nagayama, Phys. Rev. E 87, 010901 (2013).

${ }^{25}$ K. Iida, H. Kitahata, and M. Nagayama, Physica D 272, 39 (2014).

${ }^{26}$ U. Thiele, K. John, and M. Bär, Phys. Rev. Lett. 93, 027802 (2004).

${ }^{27}$ K. John, M. Bär, and U. Thiele, Eur. Phys. J. E 18, 183 (2005).

${ }^{28}$ A. Pototsky, M. Bestehorn, D. Merkt, and U. Thiele, J. Chem. Phys. 122, 224711 (2005)

${ }^{29}$ U. Thiele and E. Knobloch, Phys. Rev. Lett. 97, 204501 (2006).

${ }^{30}$ D. Shao, W. J. Rappel, and H. Levine, Phys. Rev. Lett. 105, 108104 (2010).

${ }^{31}$ U. Thiele, A. J. Archer, and M. Plapp, Phys. Fluids 24, 102107 (2012).

${ }^{32}$ G. V. Kolmakov, A. Schaefer, I. Aranson, and A. C. Balazs, Soft Matter 8, 180 (2012).

${ }^{33}$ F. Ziebert, S. Swaminathan, and I. S. Aranson, J. R. Soc., Interface 9, 1084 (2012).

${ }^{34}$ J. Toner, Y. Tu, and S. Ramaswamy, Ann. Phys. 318, 170 (2005).

${ }^{35}$ O. Schulz and M. Markus, J. Phys. Chem. B 111, 8175 (2007).

${ }^{36}$ S. Soh, K. J. M. Bishop, and B. A. Grzybowski, J. Phys. Chem. B 112, 10848 (2008).

${ }^{37}$ S. Soh, M. Branicki, and B. A. Grzybowski, J. Phys. Chem. Lett. 2, 770 (2011).

${ }^{38}$ Y. Sumino, K. H. Nagai, Y. Shikata, D. Tanaka, K. Yoshikawa, H. Chaté, and K. Oiwa, Nature 483, 448 (2012).

${ }^{39}$ G. S. Settles, Schlieren and Shadowgraph Techniques: Visualizing Phenomena in Transparent Media, Experimental Fluid Mechanics (Springer, 2001).

${ }^{40}$ J. D. Donahue and F. E. Bartell, J. Phys. Chem. 56, 480 (1952).

${ }^{41}$ P. G. de Gennes, Rev. Mod. Phys. 57, 827 (1985).

${ }^{42}$ N. M. Kovalchuk and D. Vollhardt, J. Phys. Chem. B 104, 7987 (2000).

${ }^{43}$ N. M. Kovalchuk, V. I. Kovalchuk, and D. Vollhardt, Colloids Surf., A 198-200, 223 (2002).

${ }^{44}$ T. Yamazaki, S. Omata, K. Svadlenka, and K. Ohara, Adv. Math. Sci. Appl. 16, 57 (2006).

${ }^{45} \mathrm{~K}$. Svadlenka, "Mathematical analysis and numerical computation of volume-constrained evolutionary problems, involving free boundaries," Ph.D. thesis, Graduate School of Natural Science \& Technology, Kanazawa University, 2008.

${ }^{46}$ K. Svadlenka and S. Omata, Nonlinear Anal. 69, 3202 (2008). 